
$\angle$ Research Square
Preprints are preliminary reports that have not undergone peer review.
They should not be considered conclusive, used to inform clinical practice, or referenced by the media as validated information.

\title{
The Influence of Hypoglycemia on the Specific Quality of Life in Type 2 Diabetes Mellitus: a Comparative Cross-sectional Study of Diabetics With and Without Hypoglycemia in Xi'an, China
}

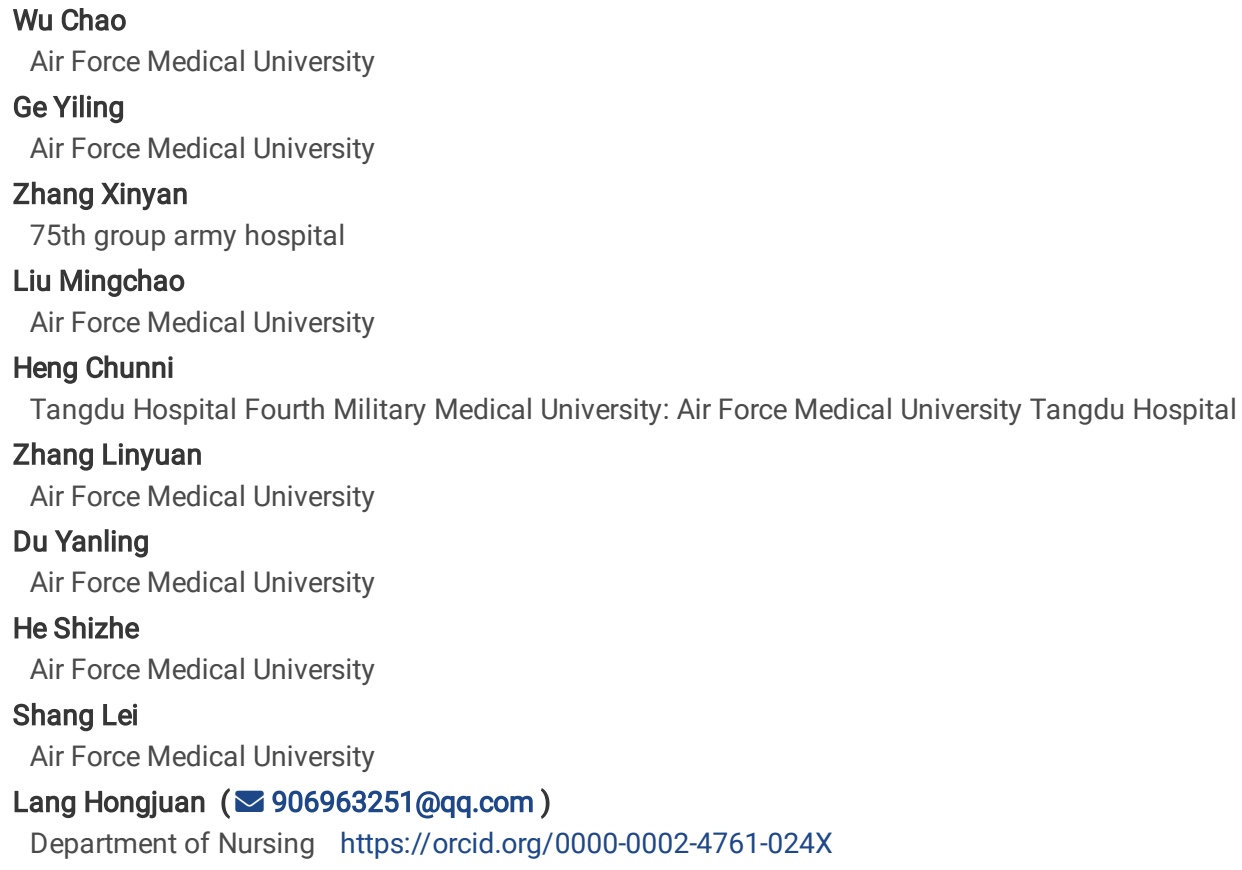




\section{Abstract}

Purpose This study aims to explore the incidence of hypoglycemia in patients with type 2 diabetes mellitus (T2DM) and the influence of hypoglycemia on the specific quality of life in T2DM patients.

Methods It was a comparative cross-sectional study consisting of 519 T2DM patients in Xi'an, China and patients were investigated by self-reported hypoglycemia and specific quality of life questionnaires during September 2019 to January 2020. Descriptive analysis, $t$-test, Chi-square test, hierarchical regression analysis and stepwise multiple regression analysis were used to assess the influence of hypoglycemia on the specific quality of life.

Results The incidence of hypoglycemia in T2DM patients was $32.18 \%$. The mean score of specific quality of life in diabetes without hypoglycemia was $57.33 \pm 15.36$ and was $61.56 \pm 17.50$ in those with hypoglycemia, which indicated that hypoglycemia had a serious impact on the quality of life diabetics $(t$ $\mathbb{Q}-5.172, p \llbracket 0.000)$. In the Univariate analysis of specific quality of life, age, education background, marital status, living status, duration of diabetes, monthly income per capita were independent and significant factors associated with specific quality of life of two groups of T2DM patients $(P<0.05)$. In the hierarchical regression analysis, the duration of the diabetes more than 11 years and the frequency of hypoglycemia more than 6 times in half a year entered the equation of specific quality of life of 519 diabetics respectively $(P<0.001)$. In multiple linear regression analysis, age, marital status and income both entered the regression equation of quality of life of the two groups $(P<0.05)$.

Conclusion Hypoglycemia will have a serious impact on the quality of life of T2DM patients. In order to improve the living quality in diabetics, effective measurements should be taken to strengthen the management of blood glucose and avoid hypoglycemia.

\section{Introduction}

As an increasingly common chronic disease, the incidence rate of Chinese diabetes is the highest in the world [1,2]. Research data shows that from 1990 to 2016, the prevalence of diabetic patients in China increased from 3.7-6.6\%, among which 15 to 49 years of age ranked the first [3]. A study of diabetes in China indicates that there were 56.4 million diabetic patients of working age in China, resulting in a loss of GDP as high as \$2.6 trillion due to the decline of productivity in 2017 [4]. Diabetes not only causes physical damage to life span and health, but also has a serious impact on individual social interaction and psychological health, which impede social and economic development $[5,6]$.

Hypoglycemia is a common symptom and a relevant burden in T2DM patients [7]. Diabetics with hypoglycemia symptoms such as dizziness, nausea, physical fatigue, often due to the overdose of oral drug and injection or insulin therapy $[8,9]$. Some studies have suggested that those diabetics with hypoglycemia always were fear of potential hypoglycemia, significantly impairing their quality of life [10-12]. In recent years, more and more attention has been paid to the psychological health and its impact on the quality of life of T2DM patients [13-15]. Systematic review studies show that the prevalence of depression in diabetes is two or three times higher than that in patients without diabetes [16]. Diabetics with hypoglycemia are more likely to suffer from anxiety, depression and other psychological problems, which will affect the control of blood sugar and treatment effect [17]. As the country with the largest number of diabetic patients, China should not only pay attention to the treatment of patients, but also the impact of the symptoms caused by diseases on their life. To further explore the effect of hypoglycemia on the quality of life of T2DM patients and its' influencing factors in China, we adopt the method of comparative cross-sectional study and quantitative analysis so as to provid reference for the comprehensive prevention and treatment of diabetes.

\section{Methods}

\section{Design}

A comparative cross-sectional study and quantitative analysis of diabetics with data collection on the basis of questionnaires.

\section{Participants}

Participants were T2DM patients from the Department of Endocrinology, Tangdu Hospital, located in the west of China. We explained the study design and the significance of research to the diabetics and reconfirmed that participants volunteered to participate in the survey and obtained their verbal and written informed consent. We used the purpose sampling method and randomly sent invitations to 580 T2DM patients during September 2019 to January 2020. After the completion of the questionnaires 519 valid questionnaires were finally confirmed.

\section{Questionnaire}

The questionnaire consists of two parts: The first part is general demographic data. It was designed by the researchers, including 9 items such as gender, age, education background, frequency of hypoglycemia (if there is no hypoglycemia, participants do not need to answer). The second part is Diabetes Specific Quality of Life Scale [18]. This scale is different from the general quality of life scale. It is combined with the condition of diabetes to investigate the physiological conditions, psychological conditions, social relations and treatment of diabetes patients. It consists of 27 items and 4 dimensions via Likert's 5-level scoring method and is widely used in Chinese diabetes patient. The higher the total score is, the more serious the damage of diabetes to the body and the worse the quality of life they will suffer. In this study, the Cronbach's alpha coefficient was 0.853 .

\section{Statistical analysis}

Statistical analysis was conducted using a commercial statistical program (SPSS for Windows, version 23.0). The measurement data are described and counted in the form of mean \pm standard deviation. The enumeration data are described and counted using frequency, percentage. Hierarchical regression 
analysis of specific quality of life consists of three models and the influencing factors of quality of life in the two groups were analyzed by multiple linear regression. All the tests were performed by two-sided test, with $P<0.05$ as the statistical difference evaluation standard.

\section{Data availability}

The questionnaire data in this study are not publicly available in order to protect the privacy of participants. It can be obtained from the corresponding author (Email:906963251@qq.com) upon reasonable request.

\section{Results}

\section{Characteristics of the participant}

A total of 519 T2DM patients participated were included in this study, 352 of whom had no hypoglycemia, while 167 had hypoglycemia before. Of the 167 with hypoglycemia, 89 had hypoglycemia once or twice in half a year; 37 had hypoglycemia three to six times in half a year; 41 had hypoglycemia more than six times in half a year. Other general demographic data are shown in Table 1. 
Table 1

General demographic data. N, number

\begin{tabular}{|c|c|c|c|c|c|c|c|}
\hline \multirow[t]{2}{*}{ Characteristics } & \multirow{2}{*}{$\begin{array}{l}\text { Total sample } \\
\mathbf{N}\end{array}$} & \multicolumn{2}{|c|}{ Diabetics without hypoglycemia } & \multicolumn{2}{|c|}{ Diabetics with hypoglycemia } & \multirow[t]{2}{*}{$\chi^{2} / U$} & \multirow[t]{2}{*}{$P$-value } \\
\hline & & $\mathbf{N}$ & $\%$ & $\mathbf{N}$ & $\%$ & & \\
\hline \multicolumn{8}{|l|}{ Sex } \\
\hline male & 329 & 228 & 69.30 & 101 & 30.70 & 0.900 & 0.343 \\
\hline Female & 190 & 124 & 65.26 & 66 & 34.74 & & \\
\hline \multicolumn{8}{|l|}{ Age } \\
\hline$\leq 20$ & 8 & 6 & 75.00 & 2 & 25.00 & -1.777 & 0.076 \\
\hline $21 \sim 40$ & 108 & 75 & 69.44 & 33 & 30.56 & & \\
\hline $41 \sim 60$ & 235 & 168 & 71.49 & 67 & 28.51 & & \\
\hline$\geq 61$ & 168 & 103 & 61.31 & 65 & 38.69 & & \\
\hline \multicolumn{8}{|l|}{ Education background } \\
\hline Primary school or below & 60 & 35 & 58.33 & 25 & 41.67 & 4.034 & 0.258 \\
\hline Junior high school & 120 & 81 & 67.50 & 39 & 32.50 & & \\
\hline Senior high school & 254 & 173 & 68.11 & 81 & 31.89 & & \\
\hline Bachelor degree or above & 85 & 63 & 74.12 & 22 & 25.88 & & \\
\hline \multicolumn{8}{|l|}{ Marital status } \\
\hline Unmarried & 49 & 34 & 69.39 & 15 & 30.61 & 3.074 & 0.215 \\
\hline Married & 408 & 282 & 69.12 & 126 & 30.88 & & \\
\hline Divorce or Bereavement & 62 & 36 & 58.06 & 26 & 41.94 & & \\
\hline \multicolumn{8}{|l|}{ Living conditions } \\
\hline Live alone & 84 & 53 & 63.10 & 31 & 36.90 & 3.090 & 0.378 \\
\hline Live with spouse & 225 & 154 & 68.44 & 71 & 31.56 & & \\
\hline Live with children & 47 & 30 & 63.83 & 17 & 36.17 & & \\
\hline Other & 163 & 115 & 70.55 & 48 & 29.45 & & \\
\hline \multicolumn{8}{|l|}{ Duration of diabetes } \\
\hline$\leq 3$ & 157 & 115 & 73.25 & 42 & 26.75 & -2.622 & 0.009 \\
\hline $4 \sim 5$ & 68 & 50 & 73.53 & 18 & 26.47 & & \\
\hline $6 \sim 10$ & 114 & 78 & 68.42 & 36 & 31.58 & & \\
\hline$\geq 11$ & 180 & 109 & 60.56 & 71 & 39.44 & & \\
\hline \multicolumn{8}{|c|}{ Monthly income per capita in family } \\
\hline$\leq 3000$ & 210 & 136 & 64.76 & 74 & 35.24 & 1.766 & 0.413 \\
\hline $3001 \sim 5000$ & 146 & 100 & 68.49 & 46 & 31.51 & & \\
\hline$\geq 5001$ & 163 & 116 & 71.17 & 47 & 28.83 & & \\
\hline \multicolumn{8}{|c|}{ Frequency of weekly exercise } \\
\hline No exercise & 67 & 44 & 65.67 & 23 & 34.33 & 0.171 & 0.982 \\
\hline $1-2$ times / week & 165 & 112 & 67.88 & 53 & 32.12 & & \\
\hline 3-5 times / week & 101 & 69 & 68.32 & 32 & 31.68 & & \\
\hline Exercise every day & 186 & 127 & 68.28 & 59 & 31.72 & & \\
\hline \multicolumn{8}{|l|}{ Frequency of hypoglycemia } \\
\hline $1-2$ times / 6 months & 89 & & & 89 & & & \\
\hline
\end{tabular}




\begin{tabular}{|c|c|c|c|c|c|}
\hline \multirow[t]{2}{*}{ Characteristics } & \multirow{2}{*}{$\begin{array}{l}\text { Total sample } \\
\text { N }\end{array}$} & Diabetics without hypoglycemia & Diabetics with hypoglycemia & \multirow{2}{*}{$x^{2} / U$} & \multirow[t]{2}{*}{$P$ value } \\
\hline & & $\mathbf{N}$ & $\%$ & & \\
\hline $3-6$ times / 6 months & 37 & & 37 & & \\
\hline More than 6 time / 6 months & 41 & & 41 & & \\
\hline
\end{tabular}

\section{Univariate analysis of specific quality of life and its four dimensions in T2DM patients with and without hypoglycemia}

As shown in Table 2, for the group of diabetics without hypoglycemia, there were statistically significant differences in specific quality of life scores with different ages, education background, marital status, living conditions, duration of diabetes, income and exercise $(P<0.05)$. For the group of diabetics with hypoglycemia, the specific quality of life scores were statistically significant in different ages, education background, marital status, living conditions, duration of diabetes, income and frequency of hypoglycemia $(P<0.05)$. 
Table 2

The Univariate analysis of specific quality of life and its four dimensions in T2DM patients

\begin{tabular}{|c|c|c|c|c|c|c|c|c|c|c|}
\hline \multirow[t]{2}{*}{ Characteristics } & \multicolumn{5}{|c|}{ Diabetics without hypoglycemia } & \multicolumn{5}{|c|}{ Diabetics with hypoglycemia } \\
\hline & $\begin{array}{l}\text { Specificity } \\
\text { quality of } \\
\text { life }\end{array}$ & $\begin{array}{l}\text { Impact on } \\
\text { physiology }\end{array}$ & $\begin{array}{l}\text { Impact on } \\
\text { psychology }\end{array}$ & $\begin{array}{l}\text { Impact } \\
\text { on } \\
\text { social } \\
\text { relations }\end{array}$ & $\begin{array}{l}\text { Impact } \\
\text { on } \\
\text { treatment }\end{array}$ & $\begin{array}{l}\text { Specificity } \\
\text { quality of } \\
\text { life }\end{array}$ & $\begin{array}{l}\text { Impact on } \\
\text { physiology }\end{array}$ & $\begin{array}{l}\text { Impact on } \\
\text { psychology }\end{array}$ & $\begin{array}{l}\text { Impact } \\
\text { on } \\
\text { social } \\
\text { relations }\end{array}$ & $\begin{array}{l}\text { Impact } \\
\text { on } \\
\text { treatment }\end{array}$ \\
\hline \multicolumn{11}{|l|}{ Sex } \\
\hline Male & $\begin{array}{l}58.07 \pm \\
14.29\end{array}$ & $\begin{array}{l}24.67 \pm \\
7.47\end{array}$ & $\begin{array}{l}20.24 \pm \\
5.13\end{array}$ & $\begin{array}{l}7.33 \pm \\
2.43\end{array}$ & $\begin{array}{l}5.83 \pm \\
1.95\end{array}$ & $\begin{array}{l}61.83 \pm \\
15.76\end{array}$ & $\begin{array}{l}26.21 \pm \\
8.13\end{array}$ & $\begin{array}{l}21.58 \pm \\
5.60\end{array}$ & $\begin{array}{l}7.51 \pm \\
2.48\end{array}$ & $\begin{array}{l}6.52 \pm \\
2.14\end{array}$ \\
\hline Female & $\begin{array}{l}55.96 \pm \\
17.12\end{array}$ & $\begin{array}{l}24.38 \pm \\
8.80\end{array}$ & $\begin{array}{l}19.03 \pm \\
6.49\end{array}$ & $\begin{array}{l}6.17 \pm \\
2.04\end{array}$ & $\begin{array}{l}5.84 \pm \\
2.11\end{array}$ & $\begin{array}{l}61.15 \pm \\
20.00\end{array}$ & $\begin{array}{l}26.91 \pm \\
10.05\end{array}$ & $\begin{array}{l}20.86 \pm \\
7.50\end{array}$ & $\begin{array}{l}6.83 \pm \\
2.26\end{array}$ & $\begin{array}{l}6.55 \pm \\
2.37\end{array}$ \\
\hline t-value & 1.233 & 0.329 & 1.92 & 2.409 & -0.044 & 0.245 & -0.496 & 0.710 & 1.798 & -0.058 \\
\hline P-value & 0.219 & 0.743 & 0.056 & 0.017 & 0.965 & 0.807 & 0.621 & 0.479 & 0.074 & 0.953 \\
\hline \multicolumn{11}{|l|}{ Age } \\
\hline$\leq 20$ & $\begin{array}{l}47.50 \pm \\
7.23\end{array}$ & $\begin{array}{l}19.33 \pm \\
4.37\end{array}$ & $\begin{array}{l}17.50 \pm \\
3.56\end{array}$ & $\begin{array}{l}5.67 \pm \\
1.37\end{array}$ & $\begin{array}{l}5.00 \pm \\
1.10\end{array}$ & $\begin{array}{l}45.50 \pm \\
2.12\end{array}$ & $\begin{array}{l}18.00 \pm \\
0.00\end{array}$ & $\begin{array}{l}16.50 \pm \\
2.12\end{array}$ & $\begin{array}{l}5.00 \pm \\
1.41\end{array}$ & $\begin{array}{l}6.00 \pm \\
1.41\end{array}$ \\
\hline $21 \sim 40$ & $\begin{array}{l}48.44 \pm \\
11.43\end{array}$ & $\begin{array}{l}18.99 \pm \\
5.28\end{array}$ & $\begin{array}{l}17.28 \pm \\
4.62\end{array}$ & $\begin{array}{l}7.13 \pm \\
2.44\end{array}$ & $\begin{array}{l}5.04 \pm \\
1.47\end{array}$ & $\begin{array}{l}46.94 \pm \\
12.53\end{array}$ & $\begin{array}{l}18.00 \pm \\
4.72\end{array}$ & $\begin{array}{l}16.55 \pm \\
5.19\end{array}$ & $\begin{array}{l}7.30 \pm \\
2.69\end{array}$ & $\begin{array}{l}5.09 \pm \\
1.57\end{array}$ \\
\hline $41 \sim 60$ & $\begin{array}{l}57.34 \pm \\
13.64\end{array}$ & $\begin{array}{l}24.64 \pm \\
6.95\end{array}$ & $\begin{array}{l}19.85 \pm \\
5.10\end{array}$ & $\begin{array}{l}7.17 \pm \\
2.23\end{array}$ & $\begin{array}{l}5.68 \pm \\
1.79\end{array}$ & $\begin{array}{l}61.95 \pm \\
14.69\end{array}$ & $\begin{array}{l}26.78 \pm \\
7.50\end{array}$ & $\begin{array}{l}21.49 \pm \\
5.52\end{array}$ & $\begin{array}{l}7.31 \pm \\
2.20\end{array}$ & $\begin{array}{l}6.37 \pm \\
1.89\end{array}$ \\
\hline$\geq 61$ & $\begin{array}{l}64.35 \pm \\
17.21\end{array}$ & $\begin{array}{l}28.83 \pm \\
8.63\end{array}$ & $\begin{array}{l}21.74 \pm \\
6.57\end{array}$ & $\begin{array}{l}7.09 \pm \\
2.41\end{array}$ & $\begin{array}{l}6.70 \pm \\
2.38\end{array}$ & $\begin{array}{l}68.87 \pm \\
17.78\end{array}$ & $\begin{array}{l}30.63 \pm \\
8.89\end{array}$ & $\begin{array}{l}23.60 \pm \\
6.57\end{array}$ & $\begin{array}{l}7.22 \pm \\
2.50\end{array}$ & $\begin{array}{l}7.42 \pm \\
2.44\end{array}$ \\
\hline F-value & 18.869 & 28.439 & 10.012 & 0.812 & 11.980 & 15.269 & 20.969 & 11.014 & 0.596 & 9.549 \\
\hline P-value & $<0.001$ & $<0.001$ & $<0.001$ & 0.488 & $<0.001$ & $<0.001$ & $<0.001$ & $<0.001$ & 0.618 & $<0.001$ \\
\hline \multicolumn{11}{|c|}{ Education background } \\
\hline $\begin{array}{l}\text { Primary } \\
\text { school or } \\
\text { below }\end{array}$ & $\begin{array}{l}67.71 \pm \\
16.27\end{array}$ & $\begin{array}{l}30.03 \pm \\
8.53\end{array}$ & $\begin{array}{l}23.06 \pm \\
6.42\end{array}$ & $\begin{array}{l}7.31 \pm \\
2.19\end{array}$ & $\begin{array}{l}7.31 \pm \\
2.72\end{array}$ & $\begin{array}{l}73.60 \pm \\
14.31\end{array}$ & $\begin{array}{l}32.72 \pm \\
7.87\end{array}$ & $\begin{array}{l}25.20 \pm \\
5.94\end{array}$ & $\begin{array}{l}7.20 \pm \\
2.22\end{array}$ & $\begin{array}{l}8.48 \pm \\
2.28\end{array}$ \\
\hline $\begin{array}{l}\text { Junior high } \\
\text { school }\end{array}$ & $\begin{array}{l}61.26 \pm \\
18.05\end{array}$ & $\begin{array}{l}27.11 \pm \\
8.65\end{array}$ & $\begin{array}{l}20.49 \pm \\
6.83\end{array}$ & $\begin{array}{l}7.51 \pm \\
2.63\end{array}$ & $\begin{array}{l}6.15 \pm \\
2.24\end{array}$ & $\begin{array}{l}66.49 \pm \\
21.34\end{array}$ & $\begin{array}{l}29.26 \pm \\
9.89\end{array}$ & $\begin{array}{l}22.49 \pm \\
7.97\end{array}$ & $\begin{array}{l}7.85 \pm \\
2.92\end{array}$ & $\begin{array}{l}6.90 \pm \\
2.48\end{array}$ \\
\hline $\begin{array}{l}\text { Senior high } \\
\text { school }\end{array}$ & $\begin{array}{l}55.14 \pm \\
13.29\end{array}$ & $\begin{array}{l}23.37 \pm \\
7.04\end{array}$ & $\begin{array}{l}19.28 \pm \\
4.98\end{array}$ & $\begin{array}{l}6.89 \pm \\
2.26\end{array}$ & $\begin{array}{l}5.60 \pm \\
1.71\end{array}$ & $\begin{array}{l}58.16 \pm \\
14.80\end{array}$ & $\begin{array}{l}24.73 \pm \\
7.85\end{array}$ & $\begin{array}{l}20.44 \pm \\
5.43\end{array}$ & $\begin{array}{l}6.99 \pm \\
2.22\end{array}$ & $\begin{array}{l}6.00 \pm \\
1.88\end{array}$ \\
\hline $\begin{array}{l}\text { Bachelor } \\
\text { degree or } \\
\text { above }\end{array}$ & $\begin{array}{l}52.51 \pm \\
12.72\end{array}$ & $\begin{array}{l}21.56 \pm \\
6.73\end{array}$ & $\begin{array}{l}18.62 \pm \\
4.62\end{array}$ & $\begin{array}{l}7.10 \pm \\
2.11\end{array}$ & $\begin{array}{l}5.24 \pm \\
1.50\end{array}$ & $\begin{array}{l}51.68 \pm \\
12.84\end{array}$ & $\begin{array}{l}20.95 \pm \\
6.46\end{array}$ & $\begin{array}{l}17.91 \pm \\
4.60\end{array}$ & $\begin{array}{l}7.18 \pm \\
2.30\end{array}$ & $\begin{array}{l}5.64 \pm \\
1.47\end{array}$ \\
\hline F-value & 11.252 & 13.988 & 5.900 & 1.402 & 10.416 & 9.626 & 10.835 & 6.698 & 1.126 & 11.157 \\
\hline P-value & $<0.001$ & $<0.001$ & 0.001 & 0.242 & $<0.001$ & $<0.001$ & $<0.001$ & $<0.001$ & 0.340 & $<0.001$ \\
\hline \multicolumn{11}{|l|}{ Marital status } \\
\hline Unmarried & $\begin{array}{l}51.41 \pm \\
12.94\end{array}$ & $\begin{array}{l}20.06 \pm \\
5.94\end{array}$ & $\begin{array}{l}18.26 \pm \\
5.14\end{array}$ & $\begin{array}{l}7.59 \pm \\
3.02\end{array}$ & $\begin{array}{l}5.50 \pm \\
1.90\end{array}$ & $\begin{array}{l}53.73 \pm \\
14.40\end{array}$ & $\begin{array}{l}20.33 \pm \\
7.01\end{array}$ & $\begin{array}{l}19.67 \pm \\
5.79\end{array}$ & $\begin{array}{l}7.73 \pm \\
2.79\end{array}$ & $\begin{array}{l}6.00 \pm \\
1.604\end{array}$ \\
\hline Married & $\begin{array}{l}56.65 \pm \\
14.71\end{array}$ & $\begin{array}{l}24.34 \pm \\
7.62\end{array}$ & $\begin{array}{l}19.53 \pm \\
5.37\end{array}$ & $\begin{array}{l}7.05 \pm \\
2.24\end{array}$ & $\begin{array}{l}5.72 \pm \\
1.93\end{array}$ & $\begin{array}{l}60.40 \pm \\
16.86\end{array}$ & $\begin{array}{l}26.06 \pm \\
8.51\end{array}$ & $\begin{array}{l}20.73 \pm \\
6.09\end{array}$ & $\begin{array}{l}7.25 \pm \\
2.38\end{array}$ & $\begin{array}{l}6.37 \pm \\
2.21\end{array}$ \\
\hline $\begin{array}{l}\text { Divorce or } \\
\text { Bereavement }\end{array}$ & $\begin{array}{l}68.22 \pm \\
17.64\end{array}$ & $\begin{array}{l}30.58 \pm \\
8.74\end{array}$ & $\begin{array}{l}23.50 \pm \\
6.95\end{array}$ & $\begin{array}{l}7.11 \pm \\
2.24\end{array}$ & $\begin{array}{l}7.03 \pm \\
2.35\end{array}$ & $\begin{array}{l}71.73 \pm \\
18.58\end{array}$ & $\begin{array}{l}32.12 \pm \\
9.04\end{array}$ & $\begin{array}{l}25.00 \pm \\
7.11\end{array}$ & $\begin{array}{l}6.96 \pm \\
2.39\end{array}$ & $\begin{array}{l}7.65 \pm \\
2.37\end{array}$ \\
\hline F-value & 12.643 & 17.366 & 9.697 & 0.806 & 7.572 & 6.582 & 9.848 & 5.620 & 0.484 & 4.228 \\
\hline P-value & $<0.001$ & $<0.001$ & $<0.001$ & 0.447 & 0.001 & 0.002 & $<0.001$ & 0.004 & 0.617 & 0.016 \\
\hline \multicolumn{11}{|l|}{ Living conditions } \\
\hline Live alone & $\begin{array}{l}62.94 \pm \\
20.24\end{array}$ & $\begin{array}{l}26.79 \pm \\
10.47\end{array}$ & $\begin{array}{l}21.75 \pm \\
7.21\end{array}$ & $\begin{array}{l}8.00 \pm \\
3.16\end{array}$ & $\begin{array}{l}6.40 \pm \\
2.58\end{array}$ & $\begin{array}{l}68.71 \pm \\
21.82\end{array}$ & $\begin{array}{l}29.84 \pm \\
11.41\end{array}$ & $\begin{array}{l}23.84 \pm \\
7.76\end{array}$ & $\begin{array}{l}7.90 \pm \\
2.88\end{array}$ & $\begin{array}{l}7.13 \pm \\
2.72\end{array}$ \\
\hline $\begin{array}{l}\text { Live with } \\
\text { spouse }\end{array}$ & $\begin{array}{l}56.19 \pm \\
14.02\end{array}$ & $\begin{array}{l}24.08 \pm \\
7.16\end{array}$ & $\begin{array}{l}19.36 \pm \\
5.19\end{array}$ & $\begin{array}{l}7.07 \pm \\
2.20\end{array}$ & $\begin{array}{l}5.68 \pm \\
1.82\end{array}$ & $\begin{array}{l}55.92 \pm \\
15.22\end{array}$ & $\begin{array}{l}25.24 \pm \\
7.51\end{array}$ & $\begin{array}{l}20.24 \pm \\
5.63\end{array}$ & $\begin{array}{l}7.25 \pm \\
2.35\end{array}$ & $\begin{array}{l}6.18 \pm \\
2.05\end{array}$ \\
\hline $\begin{array}{l}\text { Live with } \\
\text { children }\end{array}$ & $\begin{array}{l}61.83 \pm \\
16.05\end{array}$ & $\begin{array}{l}27.70 \pm \\
8.26\end{array}$ & $\begin{array}{l}21.50 \pm \\
6.16\end{array}$ & $\begin{array}{l}6.30 \pm \\
1.39\end{array}$ & $\begin{array}{l}6.33 \pm \\
2.37\end{array}$ & $\begin{array}{l}66.35 \pm \\
18.78\end{array}$ & $\begin{array}{l}29.65 \pm \\
9.65\end{array}$ & $\begin{array}{l}23.24 \pm \\
7.02\end{array}$ & $\begin{array}{l}6.06 \pm \\
1.35\end{array}$ & $\begin{array}{l}7.41 \pm \\
2.24\end{array}$ \\
\hline
\end{tabular}




\begin{tabular}{|c|c|c|c|c|c|c|c|c|c|c|}
\hline \multirow[t]{2}{*}{ Characteristics } & \multicolumn{5}{|c|}{ Diabetics without hypoglycemia } & \multicolumn{5}{|c|}{ Diabetics with hypoglycemia } \\
\hline & $\begin{array}{l}\text { Specificity } \\
\text { quality of } \\
\text { life }\end{array}$ & $\begin{array}{l}\text { Impact on } \\
\text { physiology }\end{array}$ & $\begin{array}{l}\text { Impact on } \\
\text { psychology }\end{array}$ & $\begin{array}{l}\text { Impact } \\
\text { on } \\
\text { social } \\
\text { relations }\end{array}$ & $\begin{array}{l}\text { Impact } \\
\text { on } \\
\text { treatment }\end{array}$ & $\begin{array}{l}\text { Specificity } \\
\text { quality of } \\
\text { life }\end{array}$ & $\begin{array}{l}\text { Impact on } \\
\text { physiology }\end{array}$ & $\begin{array}{l}\text { Impact on } \\
\text { psychology }\end{array}$ & $\begin{array}{l}\text { Impact } \\
\text { on } \\
\text { social } \\
\text { relations }\end{array}$ & $\begin{array}{l}\text { Impact } \\
\text { on } \\
\text { treatment }\end{array}$ \\
\hline Other & $\begin{array}{l}55.09 \pm \\
13.53\end{array}$ & $\begin{array}{l}23.37 \pm \\
7.20\end{array}$ & $\begin{array}{l}19.10 \pm \\
5.10\end{array}$ & $\begin{array}{l}6.97 \pm \\
2.11\end{array}$ & $\begin{array}{l}5.65 \pm \\
1.79\end{array}$ & $\begin{array}{l}59.17 \pm \\
15.95\end{array}$ & $\begin{array}{l}25.04 \pm \\
8.16\end{array}$ & $\begin{array}{l}20.54 \pm \\
5.89\end{array}$ & $\begin{array}{l}7.23 \pm \\
2.36\end{array}$ & $\begin{array}{l}6.35 \pm \\
2.05\end{array}$ \\
\hline F-value & 4.449 & 4.095 & 4.009 & 4.089 & 2.680 & 3.102 & 3.176 & 3.129 & 2.189 & 2.360 \\
\hline$P$-value & 0.004 & 0.007 & 0.008 & 0.007 & 0.047 & 0.028 & 0.026 & 0.027 & 0.091 & 0.073 \\
\hline \multicolumn{11}{|c|}{ Duration of diabetes } \\
\hline$\leq 3$ & $\begin{array}{l}51.39 \pm \\
12.70\end{array}$ & $\begin{array}{l}20.71 \pm \\
6.35\end{array}$ & $\begin{array}{l}18.36 \pm \\
4.94\end{array}$ & $\begin{array}{l}7.11 \pm \\
2.50\end{array}$ & $\begin{array}{l}5.21 \pm \\
1.54\end{array}$ & $\begin{array}{l}50.48 \pm \\
13.42\end{array}$ & $\begin{array}{l}20.50 \pm \\
6.61\end{array}$ & $\begin{array}{l}17.81 \pm \\
5.68\end{array}$ & $\begin{array}{l}6.88 \pm \\
2.50\end{array}$ & $\begin{array}{l}5.29 \pm \\
1.38\end{array}$ \\
\hline $4 \sim 5$ & $\begin{array}{l}56.80 \pm \\
13.41\end{array}$ & $\begin{array}{l}24.58 \pm \\
6.76\end{array}$ & $\begin{array}{l}19.50 \pm \\
4.90\end{array}$ & $\begin{array}{l}7.10 \pm \\
2.30\end{array}$ & $\begin{array}{l}5.62 \pm \\
1.81\end{array}$ & $\begin{array}{l}63.78 \pm \\
15.05\end{array}$ & $\begin{array}{l}26.78 \pm \\
7.26\end{array}$ & $\begin{array}{l}22.28 \pm \\
5.06\end{array}$ & $\begin{array}{l}7.89 \pm \\
2.63\end{array}$ & $\begin{array}{l}6.83 \pm \\
2.07\end{array}$ \\
\hline $6 \sim 10$ & $\begin{array}{l}57.95 \pm \\
14.92\end{array}$ & $\begin{array}{l}25.42 \pm \\
7.54\end{array}$ & $\begin{array}{l}19.78 \pm \\
5.97\end{array}$ & $\begin{array}{l}6.97 \pm \\
2.18\end{array}$ & $\begin{array}{l}5.79 \pm \\
1.96\end{array}$ & $\begin{array}{l}62.97 \pm \\
17.34\end{array}$ & $\begin{array}{l}27.86 \pm \\
8.65\end{array}$ & $\begin{array}{l}21.83 \pm \\
6.71\end{array}$ & $\begin{array}{l}6.81 \pm \\
1.95\end{array}$ & $\begin{array}{l}6.47 \pm \\
2.46\end{array}$ \\
\hline$\geq 11$ & $\begin{array}{l}63.39 \pm \\
16.77\end{array}$ & $\begin{array}{l}28.02 \pm \\
8.55\end{array}$ & $\begin{array}{l}21.54 \pm \\
6.08\end{array}$ & $\begin{array}{l}7.21 \pm \\
2.26\end{array}$ & $\begin{array}{l}6.61 \pm \\
2.30\end{array}$ & $\begin{array}{l}66.85 \pm \\
17.65\end{array}$ & $\begin{array}{l}29.52 \pm \\
9.09\end{array}$ & $\begin{array}{l}22.85 \pm \\
6.29\end{array}$ & $\begin{array}{l}7.52 \pm \\
2.48\end{array}$ & $\begin{array}{l}7.23 \pm \\
2.28\end{array}$ \\
\hline F-value & 12.575 & 18.584 & 6.235 & 0.157 & 10.160 & 9.111 & 10.388 & 6.310 & 1.469 & 7.585 \\
\hline P-value & $<0.001$ & $<0.001$ & $<0.001$ & 0.925 & $<0.001$ & $<0.001$ & $<0.001$ & $<0.001$ & 0.225 & $<0.001$ \\
\hline \multicolumn{11}{|c|}{ Monthly income per capita in family } \\
\hline$\leq 3000$ & $\begin{array}{l}62.27 \pm \\
17.20\end{array}$ & $\begin{array}{l}26.82 \pm \\
8.70\end{array}$ & $\begin{array}{l}21.57 \pm \\
6.33\end{array}$ & $\begin{array}{l}7.43 \pm \\
2.50\end{array}$ & $\begin{array}{l}6.46 \pm \\
2.44\end{array}$ & $\begin{array}{l}67.42 \pm \\
17.97\end{array}$ & $\begin{array}{l}29.05 \pm \\
9.08\end{array}$ & $\begin{array}{l}23.45 \pm \\
6.52\end{array}$ & $\begin{array}{l}7.45 \pm \\
2.45\end{array}$ & $\begin{array}{l}7.47 \pm \\
2.50\end{array}$ \\
\hline $3001 \sim 5000$ & $\begin{array}{l}57.54 \pm \\
15.36\end{array}$ & $\begin{array}{l}24.78 \pm \\
7.98\end{array}$ & $\begin{array}{l}19.80 \pm \\
5.56\end{array}$ & $\begin{array}{l}7.26 \pm \\
2.33\end{array}$ & $\begin{array}{l}5.70 \pm \\
1.76\end{array}$ & $\begin{array}{l}63.78 \pm \\
17.57\end{array}$ & $\begin{array}{l}28.11 \pm \\
9.02\end{array}$ & $\begin{array}{l}21.78 \pm \\
6.55\end{array}$ & $\begin{array}{l}7.48 \pm \\
2.47\end{array}$ & $\begin{array}{l}6.41 \pm \\
1.81\end{array}$ \\
\hline$\geq 5001$ & $\begin{array}{l}51.34 \pm \\
10.21\end{array}$ & $\begin{array}{l}21.74 \pm \\
5.94\end{array}$ & $\begin{array}{l}17.78 \pm \\
4.06\end{array}$ & $\begin{array}{l}6.61 \pm \\
2.01\end{array}$ & $\begin{array}{l}5.22 \pm \\
1.33\end{array}$ & $\begin{array}{l}50.17 \pm \\
9.94\end{array}$ & $\begin{array}{l}20.85 \pm \\
5.54\end{array}$ & $\begin{array}{l}17.45 \pm \\
3.98\end{array}$ & $\begin{array}{l}6.70 \pm \\
2.26\end{array}$ & $\begin{array}{l}5.17 \pm \\
1.24\end{array}$ \\
\hline F-value & 17.337 & 13.775 & 15.138 & 4.223 & 13.112 & 17.311 & 15.530 & 14.934 & 1.677 & 18.704 \\
\hline P-value & $<0.001$ & $<0.001$ & $<0.001$ & 0.015 & $<0.001$ & $<0.001$ & $<0.001$ & $<0.001$ & 0.190 & $<0.001$ \\
\hline \multicolumn{11}{|c|}{ Frequency of weekly exercise } \\
\hline No exercise & $\begin{array}{l}54.14 \pm \\
13.87\end{array}$ & $\begin{array}{l}22.55 \pm \\
7.06\end{array}$ & $\begin{array}{l}19.45 \pm \\
5.11\end{array}$ & $\begin{array}{l}6.59 \pm \\
2.38\end{array}$ & $\begin{array}{l}5.55 \pm \\
2.23\end{array}$ & $\begin{array}{l}57.52 \pm \\
16.00\end{array}$ & $\begin{array}{l}24.30 \pm \\
7.99\end{array}$ & $\begin{array}{l}20.13 \pm \\
6.01\end{array}$ & $\begin{array}{l}6.61 \pm \\
2.39\end{array}$ & $\begin{array}{l}6.48 \pm \\
2.45\end{array}$ \\
\hline $\begin{array}{l}1-2 \text { times / } \\
\text { week }\end{array}$ & $\begin{array}{l}59.70 \pm \\
17.57\end{array}$ & $\begin{array}{l}25.50 \pm \\
9.08\end{array}$ & $\begin{array}{l}20.67 \pm \\
6.30\end{array}$ & $\begin{array}{l}7.30 \pm \\
2.46\end{array}$ & $\begin{array}{l}6.22 \pm \\
2.13\end{array}$ & $\begin{array}{l}64.75 \pm \\
20.15\end{array}$ & $\begin{array}{l}27.94 \pm \\
10.13\end{array}$ & $\begin{array}{l}22.57 \pm \\
7.12\end{array}$ & $\begin{array}{l}7.32 \pm \\
2.70\end{array}$ & $\begin{array}{l}6.92 \pm \\
2.33\end{array}$ \\
\hline $\begin{array}{l}3-5 \text { times / } \\
\text { week }\end{array}$ & $\begin{array}{l}59.86 \pm \\
16.28\end{array}$ & $\begin{array}{l}25.81 \pm \\
8.13\end{array}$ & $\begin{array}{l}20.75 \pm \\
6.02\end{array}$ & $\begin{array}{l}7.06 \pm \\
2.45\end{array}$ & $\begin{array}{l}6.23 \pm \\
2.04\end{array}$ & $\begin{array}{l}66.00 \pm \\
18.76\end{array}$ & $\begin{array}{l}29.09 \pm \\
9.46\end{array}$ & $\begin{array}{l}22.91 \pm \\
6.75\end{array}$ & $\begin{array}{l}6.84 \pm \\
2.36\end{array}$ & $\begin{array}{l}7.16 \pm \\
2.30\end{array}$ \\
\hline $\begin{array}{l}\text { Exercise every } \\
\text { day }\end{array}$ & $\begin{array}{l}52.97 \pm \\
12.60\end{array}$ & $\begin{array}{l}23.77 \pm \\
6.87\end{array}$ & $\begin{array}{l}18.68 \pm \\
4.85\end{array}$ & $\begin{array}{l}7.15 \pm \\
2.09\end{array}$ & $\begin{array}{l}5.37 \pm \\
1.68\end{array}$ & $\begin{array}{l}57.86 \pm \\
13.67\end{array}$ & $\begin{array}{l}24.61 \pm \\
7.24\end{array}$ & $\begin{array}{l}19.75 \pm \\
5.29\end{array}$ & $\begin{array}{l}7.64 \pm \\
2.13\end{array}$ & $\begin{array}{l}5.86 \pm \\
1.86\end{array}$ \\
\hline F-value & 3.203 & 2.481 & 3.312 & 1.018 & 5.044 & 2.635 & 2.802 & 2.868 & 1.396 & 3.280 \\
\hline P-value & 0.023 & 0.061 & 0.020 & 0.385 & 0.002 & 0.052 & 0.042 & 0.038 & 0.246 & 0.022 \\
\hline \multicolumn{11}{|c|}{ Frequency of hypoglycemia } \\
\hline $\begin{array}{l}1-2 \text { times / } 6 \\
\text { months }\end{array}$ & & & & & & $\begin{array}{l}55.24 \pm \\
14.83\end{array}$ & $\begin{array}{l}23.39 \pm \\
7.61\end{array}$ & $\begin{array}{l}19.07 \pm \\
5.58\end{array}$ & $\begin{array}{l}7.04 \pm \\
2.40\end{array}$ & $\begin{array}{l}5.73 \pm \\
1.62\end{array}$ \\
\hline $\begin{array}{l}3-6 \text { times / } 6 \\
\text { months }\end{array}$ & & & & & & $\begin{array}{l}68.41 \pm \\
17.60\end{array}$ & $\begin{array}{l}30.30 \pm \\
9.07\end{array}$ & $\begin{array}{l}23.62 \pm \\
6.14\end{array}$ & $\begin{array}{l}7.22 \pm \\
2.39\end{array}$ & $\begin{array}{l}7.27 \pm \\
2.63\end{array}$ \\
\hline $\begin{array}{l}\text { More than } 6 \\
\text { times / } 6 \\
\text { months }\end{array}$ & & & & & & $\begin{array}{l}69.12 \pm \\
17.82\end{array}$ & $\begin{array}{l}29.76 \pm \\
9.10\end{array}$ & $\begin{array}{l}24.05 \pm \\
6.64\end{array}$ & $\begin{array}{l}7.71 \pm \\
2.44\end{array}$ & $\begin{array}{l}7.61 \pm \\
2.36\end{array}$ \\
\hline F-value & & & & & & 14.492 & 13.177 & 13.337 & 1.064 & 14.629 \\
\hline P-value & & & & & & $<0.001$ & $<0.001$ & $<0.001$ & 0.348 & $<0.001$ \\
\hline
\end{tabular}


The scores of specific quality of life of the diabetics with hypoglycemia were higher than those without hypoglycemia. And the results of $t$-test in Table 3 showed that there were significant differences in total score $(t=-5.172, P<0.001)$, dimension of impact on physiology $(t=-4.535, P<0.001)$, dimension of impact on psychology $(t=-4.916, P<0.001)$ and dimension of impact on treatment $(t=-6.523, P<0.001)$ between the two groups.

Table 3

Specific quality of life scores in T2DM patients

\begin{tabular}{|lllll|}
\hline Items & Diabetics without hypoglycemia & Diabetics with hypoglycemia & $t$-value & $P$-value \\
\hline Specificity quality of life & $57.33 \pm 15.36$ & $61.56 \pm 17.50$ & -5.172 & $<0.001$ \\
\hline Impact on physiology & $24.57 \pm 7.95$ & $26.49 \pm 8.92$ & -4.535 & $<0.001$ \\
\hline Impact on psychology & $19.82 \pm 5.67$ & $21.30 \pm 6.41$ & -4.916 & $<0.001$ \\
\hline Impact on social relations & $7.11 \pm 2.32$ & $7.25 \pm 2.41$ & -1.126 & 0.261 \\
\hline Impact on treatment & $5.83 \pm 2.01$ & $6.53 \pm 2.23$ & -6.523 & $<0.001$ \\
\hline
\end{tabular}

\section{Hierarchical regression analysis of specific quality of life of 519 T2DM patients}

In Table 4, we assigned values to independent variables. In the hierarchical regression analysis of Table 5, the results showed that income, marital status, exercise, age, gender and education background, had entered the regression equation of quality of life of 519 T2DM patients $\left(F=27.090, \mathrm{R}^{2}=0.298, P<\right.$ $0.001)$. On the basis of model 1 , the duration of diabetes was included in model $2\left(F=21.397, R^{2}=0.317, P<0.001\right)$. Hypoglycemia was included in model $3\left(\mathrm{~F}=20.004, \mathrm{R}^{2}=0.322, P<0.001\right)$.

Table 4

Assignment of independent variable

\begin{tabular}{|c|c|}
\hline Independent variable & Assignment \\
\hline Sex & Male $₫ 1 \otimes F e m a l e \rrbracket 2$ \\
\hline Age & $\leq 20 \rrbracket 000 ; 21 \sim 40 \rrbracket 001 ; 41 \sim 60 \otimes 010 ; \geq 61 \rrbracket 100$ \\
\hline Education background & 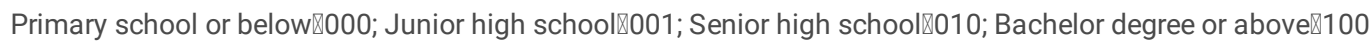 \\
\hline Marital status & Unmarried $₫ 00$; Married $₫ 01$; Divorce or bereavement $₫ 10$ \\
\hline Living conditions & Live alone $₫ 000$; Live with spouse $₫ 001$; Live with children $₫ 010$; Other $₫ 100$ \\
\hline Duration of diabetes & $\leq 3 \otimes 000 ; 4 \sim 5 \otimes 001 ; 6 \sim 10 \otimes 010 ; \geq 11 \rrbracket 100$ \\
\hline Monthly income per capita in family & $\leq 3000 \rrbracket 00 ; 3001 \sim 5000 \rrbracket 01 ; \geq 5001 \rrbracket 10$ \\
\hline Frequency of weekly exercise & No exercise $₫ 000 ; 1-2$ times / week $₫ 001 ; 3-5$ times / week $₫ 010 ;$ Exercise every day $₫ 100$ \\
\hline Frequency of hypoglycemia & 1-2 times / 6 months $₫ 00$; 3-6 times / 6 months $₫ 01$; More than 6 times / 6 months $₫ 10$ \\
\hline
\end{tabular}


Table 5

Hierarchical regression analysis of specific quality of life of T2DM patients. ${ }^{*} P<0.05,{ }^{* \star} P<0.01$, ${ }^{* \star *} P<0.001$

\begin{tabular}{|c|c|c|c|}
\hline \multirow[t]{2}{*}{ Independent variable } & \multicolumn{3}{|c|}{ Specificity quality of life diabetics } \\
\hline & M1 & M2 & M3 \\
\hline Monthly income per capita in family $\geq 5001$ yuan & $-0.293^{\star \star \star}$ & $-0.296^{\star \star \star}$ & $-0.293^{\star \star \star}$ \\
\hline Divorce or bereavement & $0.139^{\star \star}$ & $0.127^{\star \star}$ & $0.125^{\star \star}$ \\
\hline Exercise every day & $-0.133^{\star \star}$ & $-0.154^{\star \star \star}$ & $-0.153^{\star \star \star}$ \\
\hline$\geq 61$ years old & $0.430^{\star \star *}$ & $0.299^{\star \star \star}$ & $0.300^{\star \star \star}$ \\
\hline $41 \sim 60$ years old & $0.319^{\star \star \star}$ & $0.239^{\star \star \star}$ & $0.244^{\star \star \star}$ \\
\hline Monthly income per capita in family: $3000-5000$ yuan & $-0.110^{\star \star}$ & $-0.108^{\star \star}$ & $-0.107^{\star}$ \\
\hline Sex & $-0.125^{\star \star}$ & $-0.114^{\star \star}$ & $-0.116^{\star \star}$ \\
\hline Education background: Senior high school & $-0.083^{*}$ & $-0.092^{*}$ & $-0.093^{*}$ \\
\hline Duration of diabetes $4 \sim 5$ years & & 0.062 & 0.061 \\
\hline Duration of diabetes $6 \sim 10$ years & & 0.082 & 0.078 \\
\hline Duration of diabetes $\geq 11$ years & & $0.207^{\star \star \star}$ & $0.198^{\star \star}$ \\
\hline Hypoglycemia or not & & & -0.070 \\
\hline $\mathrm{R}^{2}$ & 0.298 & 0.317 & 0.322 \\
\hline$\Delta \mathrm{R}^{2}$ & & 0.019 & 0.005 \\
\hline $\mathrm{F}$ & $27.090^{\star \star \star}$ & $21.397^{\star \star \star}$ & $20.004^{\star \star \star}$ \\
\hline
\end{tabular}

\section{Stepwise multiple linear regression analysis of specific quality of life of diabetics with and without hypoglycemia}

For the group without hypoglycemia, the results of stepwise multiple linear regression analysis in Table 6 showed that gender, age, marital status, duration of diabetes, income, exercise were the influencing factors for the specific quality of life $(F=17.360, P=0.000)$. For diabetics with hypoglycemia, age, marital status, income and frequency of hypoglycemia were the influencing factors for the specific quality of life $(F=19.420, P=0.000)$. 
Table 6

Stepwise multiple linear regression analysis of specific quality of life of T2DM patients. ${ }^{\star} P<0.05,{ }^{* \star} P<0.01,{ }^{\star \star \star} P<0.001$

\begin{tabular}{|c|c|c|c|c|c|c|}
\hline Personnel sorts & Independent variable & $\begin{array}{l}\text { Specificity } \\
\text { quality of life }\end{array}$ & $\begin{array}{l}\text { Impact on } \\
\text { physiology }\end{array}$ & $\begin{array}{l}\text { Impact on } \\
\text { psychology }\end{array}$ & $\begin{array}{l}\text { Impact on social } \\
\text { relations }\end{array}$ & $\begin{array}{l}\text { Impact on } \\
\text { treatment }\end{array}$ \\
\hline \multirow{12}{*}{$\begin{array}{l}\text { Diabetics without } \\
\text { hypoglycemia }\end{array}$} & Sex & $-0.119^{*}$ & & $-0.165^{\star \star}$ & $-0.154^{\star \star}$ & \\
\hline & $21 \sim 40$ years old & $-0.221^{\star \star \star}$ & $-0.269^{\star * *}$ & $-0.165^{\star *}$ & & \\
\hline & $41 \sim 60$ years old & & & & & $0.210^{\star *}$ \\
\hline & $\geq 61$ years old & & $0.135^{\star}$ & & & $0.304^{\star \star \star}$ \\
\hline & Married & & & & & $-0.114^{*}$ \\
\hline & Divorce or bereavement & $0.163^{\star \star}$ & $0.127^{\star \star}$ & $0.161^{\star *}$ & & \\
\hline & Duration of diabetes $\geq 11$ years & $0.188^{\star \star \star}$ & $0.154^{\star *}$ & $0.146^{\star \star \star}$ & & $0.189^{\star *}$ \\
\hline & $\begin{array}{l}\text { Monthly income per capita } \\
\text { in family: } 3000-5000 \text { yuan }\end{array}$ & $-0.120^{*}$ & & $-0.135^{*}$ & & $-0.130^{*}$ \\
\hline & $\begin{array}{l}\text { Monthly income per capita in family } \\
\geq 5001 \text { yuan }\end{array}$ & $-0.291^{\star \star \star}$ & $-0.167^{\star \star \star}$ & $-0.288^{\star \star \star}$ & $-0.174^{\star \star}$ & $-0.218^{\star \star \star}$ \\
\hline & Exercise once or twice a week & & $0.174^{\star \star}$ & & & \\
\hline & Exercise three to five times a week & & $0.100^{\star}$ & & & \\
\hline & Exercise every day & $-0.167^{\star *}$ & & $-0.186^{* * *}$ & & $-0.227^{\star \star \star}$ \\
\hline $\mathrm{R}^{2}$ & & 0.261 & 0.286 & 0.222 & 0.046 & 0.224 \\
\hline F-value & & $17.360^{\star \star \star}$ & $19.682^{\star \star \star}$ & $13.994^{\star \star \star}$ & $8.398^{\star \star \star}$ & $14.179^{\star \star \star}$ \\
\hline \multirow{12}{*}{$\begin{array}{l}\text { Diabetics with } \\
\text { Hypoglycemia }\end{array}$} & $21 \sim 40$ years old & $-0.337^{\star \star \star}$ & $-0.331^{\star \star \star}$ & $-0.303^{\star \star *}$ & & \\
\hline & $41 \sim 60$ years old & & & & & $0.261^{\star *}$ \\
\hline & $\geq 61$ years old & & 0.120 & & & $0.355^{\star \star \star}$ \\
\hline & Junior high school & & & & $0.176^{*}$ & \\
\hline & Senior high school & & $-0.128^{*}$ & & & \\
\hline & Married & $-0.143^{*}$ & & $-0.178^{\star \star}$ & & \\
\hline & Live with children & & & & $-0.199^{*}$ & \\
\hline & $\begin{array}{l}\text { Monthly income per capita } \\
\text { in family: } 3000-5000 \text { yuan }\end{array}$ & & & & & $-0.219^{\star *}$ \\
\hline & $\begin{array}{l}\text { Monthly income per capita in family } \\
\geq 5001 \text { yuan }\end{array}$ & $-0.272^{\star \star \star}$ & $-0.240^{\star \star \star}$ & $-0.246^{\star \star *}$ & & $-0.324^{\star \star \star}$ \\
\hline & Exercise every day & & & & & $-0.159^{*}$ \\
\hline & $\begin{array}{l}\text { Hypoglycemia 3-6 times / } 6 \\
\text { months }\end{array}$ & $0.208^{\star *}$ & $0.178^{\star \star}$ & $0.203^{\star *}$ & & $0.153^{*}$ \\
\hline & $\begin{array}{l}\text { Hypoglycemia occurred more than } 6 \\
\text { times / } 6 \text { months }\end{array}$ & $0.263^{\star \star \star}$ & $0.221^{\star \star}$ & $0.260^{\star \star \star}$ & & $0.304^{\star \star \star}$ \\
\hline \multicolumn{2}{|l|}{$\mathrm{R}^{2}$} & 0.376 & 0.409 & 0.336 & 0.057 & 0.378 \\
\hline \multicolumn{2}{|l|}{ F-value } & $19.420^{\star \star \star}$ & $18.478^{\star \star \star}$ & $16.290^{\star \star \star}$ & $4.987^{\star \star}$ & $13.825^{\star \star \star}$ \\
\hline
\end{tabular}

\section{Discussion}

As the world's highest incidence of chronic non-communicable diseases, diabetes is the leading cause of major complications, such as end-stage renal disease and lower extremity amputations [19]. The findings of our study revealed the poor overall living quality of T2DM patients in China, and the incidence of hypoglycemia in Chinese T2DM patients was $32.18 \%$ which was higher than the proportion of patients reporting hypoglycemic symptoms in Thailand (30.65\%) [20] and lower than that in the United States (63\%) [21]. There were 167 diabetics with hypoglycemia with the specific quality of life 
score being $61.56 \pm 17.50$ and 352 diabetics without hypoglycemia with the specific quality of life score being $57.33 \pm 15.36$, which both were in a relatively high score level.

Through statistical analysis, we found the living quality of those elderly, low education, low income, divorced or widowed, living alone, long duration of diabetes were worse and lower which was consistent with the findings of India and Nepal [22, 23]. As the population ages and the chronic disease progresses, the number of elderly diabetics will continue to rise. Besides, due to the declines in body functions, vulnerabilities from comorbidities, psychological factors and geriatric syndromes such as frailty and cognitive impairment, the heavy multidimensional burden seriously diminished those elderly diabetics' living quality [24]. Moreover, with the progression of the diabetes, the large physical, psychological and financial burden deriving from diabetes' complications and treatment also increased. So the long duration of diabetes prominently diminished the specific quality of life [25]. Studies found that in those with diabetes, improvement in physical activity levels offers cardiometabolic, kidney, and cognitive benefits [24]. Exercise could enhance cardiovascular system function and physical feeling, improve insulin sensitivity, relieve blood pressure, blood lipids, and hence improve the effect of treatment. Therefore, nowadays, most expert guidelines have recommended or adopted a comprehensive chronic care model and precision diabetes medicine to optimize the diagnosis, prediction, prevention and treatment of diabetes by integrating multidimensional evaluation and multidisciplinary panel [26-28].

The results of t-test in Table 2 showed that compared with those without hypoglycemia, the score of specific quality of life in diabetes with hypoglycemia was higher $(P<0.001)$. Besides, the variable of hypoglycemia also was included in the model 3 of hierarchical regression analysis in Table 5 which indicated that hypoglycemia could impaired the specific quality of life. Hypoglycemia is potentially one of the most severe acute adverse effects of therapies for diabetes. Elliott L [29] reported that the incidence rates of severe hypoglycemia in T2DM patients the incidence rates ranged from 0 to 20 per 100 person-years. There is no doubt that hypoglycemia puts patients at risk for falls, fall-related injuries [30], dementia and death. Non-severe hypoglycemia events usually generate autonomic and neuroglycopenic symptoms which enable the individual to identify the onset, and to treat the falling blood glucose without requiring assistance while severe hypoglycemia is associated with impaired cognitive and physical functioning and the progressive neuroglycopenia interferes with the ability to self-treat [31]. Hypoglycemia and fear of hypoglycemia may further reduce adherence to glucose-lowering regimens, contributing to the further aggravation of diabetes-related complications. Therefore, it has a significant adverse impact on quality-of-life measures in diabetes [32,33], which also supported our study's finding. Psychologically, patients with recurrent hypoglycemia have been found to have chronic mood disorders including depression and anxiety; nocturnal hypoglycemia in particular may impact one's sense of well-being on the following day because of its impact on sleep quantity and quality [27]. In the realistic scene, when diabetics have hypoglycemia, they will have a sense of near death in serious cases and if they are not treated in a short time, they will cause irreversible brain damage or even death, which scary them severely [34, 35]. Most of the time, the occurrence of hypoglycemia is not easy to detect, namely asymptomatic hypoglycemia [36]. Diabetics who have suffered from hypoglycemia will always worry that they will get hypoglycemia again [37].In the future blood glucose management, diabetics dare not make their blood glucose too low, causing a vicious circle of poor management of blood glucose and thus damage their quality of life[20, 38, 39].

\section{Conclusions}

With the development of aging society in China, the number and burden of diabetes are increasing [40]. Health China 2030 program proposes to improve the health level of the whole people [41]. Therefore, given that diabetes being with such high incidence rate and mortality worldwide, especially in China [42, 43] and hypoglycemia had such serious adverse effects on the diabetics' living quality, effective approaches must been taken, particularly the measurements of decreasing the risk of iatrogenic hypoglycemia. On the one hand, medical staff should carry out health education on diabetes [44], which can learn from Karamanakos's [45] advocacy of community-based intervention for diabetes to carry out one-to-one guidance to strengthen blood glucose management and reduce the incidence of hypoglycemia. On the other hand, doctors should strengthen the screening of anxiety and depression in diabetics [46]. Studies found that early detection of anxiety and depression can significantly improve the quality of life of diabetics [47]. Besides, for patients, proper yoga and exercise [48,49], increasing peer support and company can also alleviate depression, improve the quality of life of diabetics [50]. Diabetics can broaden their social contact by closely connecting with other patients and sharing their disease experience. In particularly for those with hypoglycemia, it is advisable to seek external help to deal with negative events and increase the level of resilience [51].

\section{Declarations}

\section{Acknowledgements:}

The authors gratefully thank the nurses of the Department of Endocrinology, Tangdu Hospital for helping to collect the questionnaires. Thanks to all the participants for the contribution to this study.

\section{Ethical Approval and Consent to participate:}

This study was approved by the ethics committee of Tangdu Hospital (TDLL2018-03-88). Informed consent was obtained from all individual participants included in the study.

\section{Consent for publication:}

All the authors agreed to publish.

\section{Availability of supporting data:}


The supporting data can be obtained by contacting the corresponding author by email ( $906963251 @ q q . c o m$ ).

\section{Competing interests:}

No conflict of interest.

\section{Funding:}

None.

\section{Authors' contributions:}

All the authors contributed to the design, analysis and writing of the paper.

\section{References}

1. Dagenais, G. R., Leong, D. P., Rangarajan, S., Lanas, F., Lopez-Jaramillo, P., Gupta, R., et al. (2020). Variations in common diseases, hospital admissions, and deaths in middle-aged adults in 21 countries from five continents (PURE): a prospective cohort study. Lancet, 395(10226), 785-794, doi:10.1016/s0140-6736(19)32007-0.

2. Yang, L., Shao, J., Bian, Y., Wu, H., Shi, L., Zeng, L., et al. (2016). Prevalence of type 2 diabetes mellitus among inland residents in China (2000-2014): A meta-analysis. J Diabetes Investig, 7(6), 845-852, doi:10.1111/jdi.12514.

3. Liu, M., Liu, S. W., Wang, L. J., Bai, Y. M., Zeng, X. Y., Guo, H. B., et al. (2019). Burden of diabetes, hyperglycaemia in China from to 2016: Findings from the 1990 to 2016, global burden of disease study. Diabetes Metab, 45(3), 286-293, doi:10.1016/j.diabet.2018.08.008.

4. Hird, T. R., Zomer, E., Owen, A., Chen, L., Ademi, Z., Magliano, D. J., et al. (2019). The impact of diabetes on productivity in China. Diabetologia, 62(7), 1195-1203, doi:10.1007/s00125-019-4875-4.

5. Helgeson, V. S., Orchard, T. J., Seltman, H., Becker, D., \& Libman, I. (2019). Psychosocial predictors of diabetes risk factors and complications: An 11year follow-up. Health Psychol, 38(7), 567-576, doi:10.1037/hea0000730.

6. Gonder-Frederick, L. A., Shepard, J. A., Grabman, J. H., \& Ritterband, L. M. (2016). Psychology, technology, and diabetes management. Am Psychol, 71(7), 577-589, doi:10.1037/a0040383.

7. DeCarlo, K., \& Wallia, A. (2019). Inpatient Management of T2DM and Hyperglycemia in Older Adults. Curr Diab Rep, 19(10), 104, doi:10.1007/s11892019-1209-3.

8. Umpierrez, G., \& Korytkowski, M. (2016). Diabetic emergencies - ketoacidosis, hyperglycaemic hyperosmolar state and hypoglycaemia. Nat Rev Endocrinol, 12(4), 222-232, doi:10.1038/nrendo.2016.15.

9. Jabbour, S., Seufert, J., Scheen, A., Bailey, C. J., Karup, C., \& Langkilde, A. M. (2018). Dapagliflozin in patients with type 2 diabetes mellitus: A pooled analysis of safety data from phase Ilb/III clinical trials. Diabetes Obes Metab, 20(3), 620-628, doi:10.1111/dom.13124.

10. Rossi, M. C., Nicolucci, A., Ozzello, A., Gentile, S., Aglialoro, A., Chiambretti, A., et al. (2019). Impact of severe and symptomatic hypoglycemia on quality of life and fear of hypoglycemia in type 1 and type 2 diabetes. Results of the Hypos-1 observational study. Nutr Metab Cardiovasc Dis, 29(7), 736-743, doi:10.1016/j.numecd.2019.04.009.

11. Driscoll, K. A., Raymond, J., Naranjo, D., \& Patton, S. R. (2016). Fear of Hypoglycemia in Children and Adolescents and Their Parents with Type 1 Diabetes. Curr Diab Rep, 16(8), 77, doi:10.1007/s11892-016-0762-2.

12. Jing, X., Chen, J., Dong, Y., Han, D., Zhao, H., Wang, X., et al. (2018). Related factors of quality of life of type 2 diabetes patients: a systematic review and meta-analysis. Health Qual Life Outcomes, 16(1), 189, doi:10.1186/s12955-018-1021-9.

13. Snoek, F. J., Bremmer, M. A., \& Hermanns, N. (2015). Constructs of depression and distress in diabetes: time for an appraisal. Lancet Diabetes Endocrinol, 3(6), 450-460, doi:10.1016/s2213-8587(15)00135-7.

14. Buchberger, B., Huppertz, H., Krabbe, L., Lux, B., Mattivi, J. T., \& Siafarikas, A. (2016). Symptoms of depression and anxiety in youth with type 1 diabetes: A systematic review and meta-analysis. Psychoneuroendocrinology, 70, 70-84, doi:10.1016/j.psyneuen.2016.04.019.

15. Henríquez-Tejo, R., \& Cartes-Velásquez, R. (2018). [Psychosocial impact of type 1 diabetes mellitus in children, adolescents and their families. Literature review]. Rev Chil Pediatr, 89(3), 391-398, doi:10.4067/s0370-41062018005000507.

16. Roy, T., \& Lloyd, C. E. (2012). Epidemiology of depression and diabetes: a systematic review. J Affect Disord, 142 Suppl, S8-21, doi:10.1016/s01650327(12)70004-6.

17. Kostov, K. (2019). Effects of Magnesium Deficiency on Mechanisms of Insulin Resistance in Type 2 Diabetes: Focusing on the Processes of Insulin Secretion and Signaling. Int J Mol Sci, 20(6), doi:10.3390/ijms20061351.

18. Lee, E. H., Lee, Y. W., Lee, K. W., Kim, D. J., \& Kim, S. K. (2012). Development and psychometric evaluation of a diabetes-specific quality-of-life (D-QOL) scale. Diabetes Res Clin Pract, 95(1), 76-84, doi:10.1016/j.diabres.2011.08.022.

19. Dong, D., Lou, P., Wang, J., Zhang, P., Sun, J., Chang, G., et al. (2020). Interaction of sleep quality and anxiety on quality of life in individuals with type 2 diabetes mellitus. Health Qual Life Outcomes, 18(1), 150, doi:10.1186/s12955-020-01406-Z.

20. Pratipanawatr, T., Satirapoj, B., Ongphiphadhanakul, B., Suwanwalaikorn, S., \& Nitiyanant, W. (2019). Impact of Hypoglycemia on Health-Related Quality of Life among Type 2 Diabetes: A Cross-Sectional Study in Thailand. J Diabetes Res, 2019, 5903820, doi:10.1155/2019/5903820. 
21. Marrett, E., Radican, L., Davies, M. J., \& Zhang, Q. (2011). Assessment of severity and frequency of self-reported hypoglycemia on quality of life in patients with type 2 diabetes treated with oral antihyperglycemic agents: A survey study. BMC Res Notes, 4, 251, doi:10.1186/1756-0500-4-251.

22. John, R., Pise, S., Chaudhari, L., \& Deshpande, P. R. (2019). Evaluation of Quality of Life in Type 2 Diabetes Mellitus Patients Using Quality of Life Instrument for Indian Diabetic Patients: A Cross-Sectional Study. J Midlife Health, 10(2), 81-88, doi:10.4103/jmh.JMH_32_18.

23. Thapa, S., Pyakurel, P., Baral, D. D., \& Jha, N. (2019). Health-related quality of life among people living with type 2 diabetes: a community based crosssectional study in rural Nepal. BMC Public Health, 19(1), 1171, doi:10.1186/s12889-019-7506-6.

24. Charleer, S., De Block, C., Van Huffel, L., Broos, B., Fieuws, S., Nobels, F., et al. (2020). Quality of Life and Glucose Control After 1 Year of Nationwide Reimbursement of Intermittently Scanned Continuous Glucose Monitoring in Adults Living With Type 1 Diabetes (FUTURE): A Prospective Observational Real-World Cohort Study. Diabetes Care, 43(2), 389-397, doi:10.2337/dc19-1610.

25. de Boer, I. H., Caramori, M. L., Chan, J. C. N., Heerspink, H. J. L., Hurst, C., Khunti, K., et al. (2020). TEMPORARY REMOVAL: Executive summary of the 2020 KDIGO Diabetes Management in CKD Guideline: Evidence-based advances in monitoring and treatment. Kidney Int, doi:10.1016/j.kint.2020.06.024.

26. Chung, W. K., Erion, K., Florez, J. C., Hattersley, A. T., Hivert, M. F., Lee, C. G., et al. (2020). Precision Medicine in Diabetes: A Consensus Report From the American Diabetes Association (ADA) and the European Association for the Study of Diabetes (EASD). Diabetes Care, 43(7), 1617-1635, doi:10.2337/dci20-0022 .

27. Sayyed Kassem, L., \& Aron, D. C. (2020). The assessment and management of quality of life of older adults with diabetes mellitus. Expert Rev Endocrinol Metab, 15(2), 71-81, doi:10.1080/17446651.2020.1737520.

28. Conlin, P. R., Colburn, J., Aron, D., Pries, R. M., Tschanz, M. P., \& Pogach, L. (2017). Synopsis of the 2017 U.S. Department of Veterans Affairs/U.S. Department of Defense Clinical Practice Guideline: Management of Type 2 Diabetes Mellitus. Ann Intern Med, 167(9), 655-663, doi:10.7326/M17-1362.

29. Elliott, L., Fidler, C., Ditchfield, A., \& Stissing, T. (2016). Hypoglycemia Event Rates: A Comparison Between Real-World Data and Randomized Controlled Trial Populations in Insulin-Treated Diabetes. Diabetes Ther, 7(1), 45-60, doi:10.1007/s13300-016-0157-z.

30. Rasmussen, N. H., Dal, J., den Bergh, J. V., de Vries, F., Jensen, M. H., \& Vestergaard, P. (2020). Increased risk of falls, fall-related injuries and fractures in people with type 1 and type 2 diabetes - a nationwide cohort study. Curr Drug Saf, doi:10.2174/1574886315666200908110058.

31. Thieu, V. T., Mitchell, B. D., Varnado, O. J., \& Frier, B. M. (2020). Treatment and prevention of severe hypoglycaemia in people with diabetes: Current and new formulations of glucagon. Diabetes Obes Metab, 22(4), 469-479, doi:10.1111/dom.13941.

32. R, S. E., John, A., Belinda, C., Philip, C., Samuel, D.-J., Lisa, F., et al. (2013). Hypoglycemia and diabetes: a report of a workgroup of the American Diabetes Association and the Endocrine Society. \%J Diabetes care. 36(5).

33. D, F. E., D, B. N., D, F. I., L, E. T., G, H. L., \& Rodolfo, A. (2018). Improved Health-Related Quality of Life in a Phase 3 Islet Transplantation Trial in Type 1 Diabetes Complicated by Severe Hypoglycemia. \%J Diabetes care. 41(5).

34. Yun, J. S., \& Ko, S. H. (2015). Avoiding or coping with severe hypoglycemia in patients with type 2 diabetes. Korean J Intern Med, 30(1), 6-16, doi:10.3904/kjim.2015.30.1.6.

35. Martyn-Nemeth, P., Quinn, L., Penckofer, S., Park, C., Hofer, V., \& Burke, L. (2017). Fear of hypoglycemia: Influence on glycemic variability and selfmanagement behavior in young adults with type 1 diabetes. J Diabetes Complications, 31(4), 735-741, doi:10.1016/j.jdiacomp.2016.12.015.

36. Szadkowska, A., Czyżewska, K., Pietrzak, I., Mianowska, B., Jarosz-Chobot, P., \& Myśliwiec, M. (2018). Hypoglycaemia unawareness in patients with type 1 diabetes. Pediatr Endocrinol Diabetes Metab, 2018(3), 126-134, doi:10.5114/pedm.2018.80994.

37. Castellano-Guerrero, A. M., Guerrero, R., Relimpio, F., Losada, F., Mangas, M. A., Pumar, A., et al. (2018). Prevalence and predictors of depression and anxiety in adult patients with type 1 diabetes in tertiary care setting. Acta Diabetol, 55(9), 943-953, doi:10.1007/s00592-018-1172-5.

38. Rechenberg, K., Whittemore, R., \& Grey, M. (2017). Anxiety in Youth With Type 1 Diabetes. J Pediatr Nurs, 32, 64-71, doi:10.1016/j.pedn.2016.08.007.

39. Shiu, A. T., Thompson, D. R., \& Wong, R. Y. (2008). Quality of life and its predictors among Hong Kong Chinese patients with diabetes. J Clin Nurs, 17(5a), 125-132, doi:10.1111/j.1365-2702.2007.02036.x.

40. Fitzmaurice, C., Abate, D., Abbasi, N., Abbastabar, H., Abd-Allah, F., Abdel-Rahman, O., et al. (2019). Global, Regional, and National Cancer Incidence, Mortality, Years of Life Lost, Years Lived With Disability, and Disability-Adjusted Life-Years for 29 Cancer Groups, 1990 to 2017 : A Systematic Analysis for the Global Burden of Disease Study. JAMA Oncol, 5(12), 1749-1768, doi:10.1001/jamaoncol.2019.2996.

41. Ning, P., Schwebel, D. C., \& Hu, G. (2017). Healthy China 2030: a missed opportunity for injury control. Inj Prev, 23(6), 363, doi:10.1136/injuryprev-2017042314.

42. Rodríguez-Almagro, J., García-Manzanares, Á., Lucendo, A. J., \& Hernández-Martínez, A. (2018). Health-related quality of life in diabetes mellitus and its social, demographic and clinical determinants: A nationwide cross-sectional survey. J Clin Nurs, 27(21-22), 4212-4223, doi:10.1111/jocn.14624.

43. Fung, C. S., Wan, E. Y., Chan, A. K., \& Lam, C. L. (2017). Association of estimated glomerular filtration rate and urine albumin-to-creatinine ratio with incidence of cardiovascular diseases and mortality in chinese patients with type 2 diabetes mellitus - a population-based retrospective cohort study. BMC Nephrol, 18(1), 47, doi:10.1186/s12882-017-0468-y.

44. Pintus, D., \& Ng, S. M. (2019). Freestyle libre flash glucose monitoring improves patient quality of life measures in children with Type 1 diabetes mellitus (T1DM) with appropriate provision of education and support by healthcare professionals. Diabetes Metab Syndr, 13(5), 2923-2926, doi:10.1016/j.dsx.2019.07.054.

45. Karamanakos, G., Costa-Pinel, B., Gilis-Januszewska, A., Velickiene, D., Barrio-Torrell, F., Cos-Claramunt, X., et al. (2019). The effectiveness of a community-based, type 2 diabetes prevention programme on health-related quality of life. The DE-PLAN study. PLoS One, 14(10), e0221467,

Page 13/14 
doi:10.1371/journal.pone.0221467.

46. Bădescu, S. V., Tătaru, C., Kobylinska, L., Georgescu, E. L., Zahiu, D. M., Zăgrean, A. M., et al. (2016). The association between Diabetes mellitus and Depression. J Med Life, 9(2), 120-125.

47. Liu, X., Haagsma, J., Sijbrands, E., Buijks, H., Boogaard, L., Mackenbach, J. P., et al. (2020). Anxiety and depression in diabetes care: longitudinal associations with health-related quality of life. Sci Rep, 10(1), 8307, doi:10.1038/s41598-020-57647-x.

48. Shiju, R., Thomas, D., Al Arouj, M., Sharma, P., Tuomilehto, J., \& Bennakhi, A. (2019). Effect of Sudarshan Kriya Yoga on anxiety, depression, and quality of life in people with type 2 diabetes: A pilot study in Kuwait. Diabetes Metab Syndr, 13(3), 1995-1999, doi:10.1016/j.dsx.2019.04.038.

49. Lai, Y. C., Liao, Y. M., \& Lin, P. C. (2019). [Physical Activity and Quality of Life in Patients With Type II Diabetes Mellitus]. Hu Li Za Zhi, 66(2), 57-66, doi:10.6224/jn.201904_66(2).08.

50. Cherrington, A. L., Khodneva, Y., Richman, J. S., Andreae, S. J., Gamboa, C., \& Safford, M. M. (2018). Impact of Peer Support on Acute Care Visits and Hospitalizations for Individuals With Diabetes and Depressive Symptoms: A Cluster-Randomized Controlled Trial. Diabetes Care, 41(12), 2463-2470, doi:10.2337/dc18-0550.

51. Luo, D., Xu, J. J., Cai, X., Zhu, M., Wang, H., Yan, D., et al. (2019). The effects of family functioning and resilience on self-management and glycaemic control among youth with type 1 diabetes. J Clin Nurs, 28(23-24), 4478-4487, doi:10.1111/jocn.15033. 